\title{
Correction to: Morphological characteristics of chronic total occlusion: predictors of different strategies for long-segment femoral arterial occlusions
}

\author{
Li-Ming Wei ${ }^{1}$ Yue-Qi Zhu ${ }^{1}$ Pei-Lei Zhang ${ }^{1} \cdot$ Fang Liu $^{2} \cdot$ Hai-Tao Lu ${ }^{1}$. \\ Jun-Gong Zhao ${ }^{1}$
}

Published online: 9 January 2018

(C) European Society of Radiology 2018

\section{Correction to: Eur Radiol}

https://doi.org/10.1007/s00330-017-5003-9

The original version of this article, published on 21 August 2017, unfortunately contained a mistake. The following correction has therefore been made in the original:

The legends to Fig. 2-4 were interchanged. The correct versions are given below. The original article has been corrected.

The online version of the original article can be found at https://doi.org/ 10.1007/s00330-017-5003-9

Hai-Tao Lu

luhaitao1975@126.com

$\triangle$ Jun-Gong Zhao

zhaojungongradio@hotmail.com

1 Department of Diagnostic and Interventional Radiology, Shanghai Jiaotong University Affiliated Sixth People's Hospital, No. 600, Yi Shan Road, Shanghai 200233, China

2 Department of Endocrinology, Shanghai Jiaotong University Affiliated Sixth People's Hospital, No. 600, Yi Shan Road, Shanghai 200233, China 

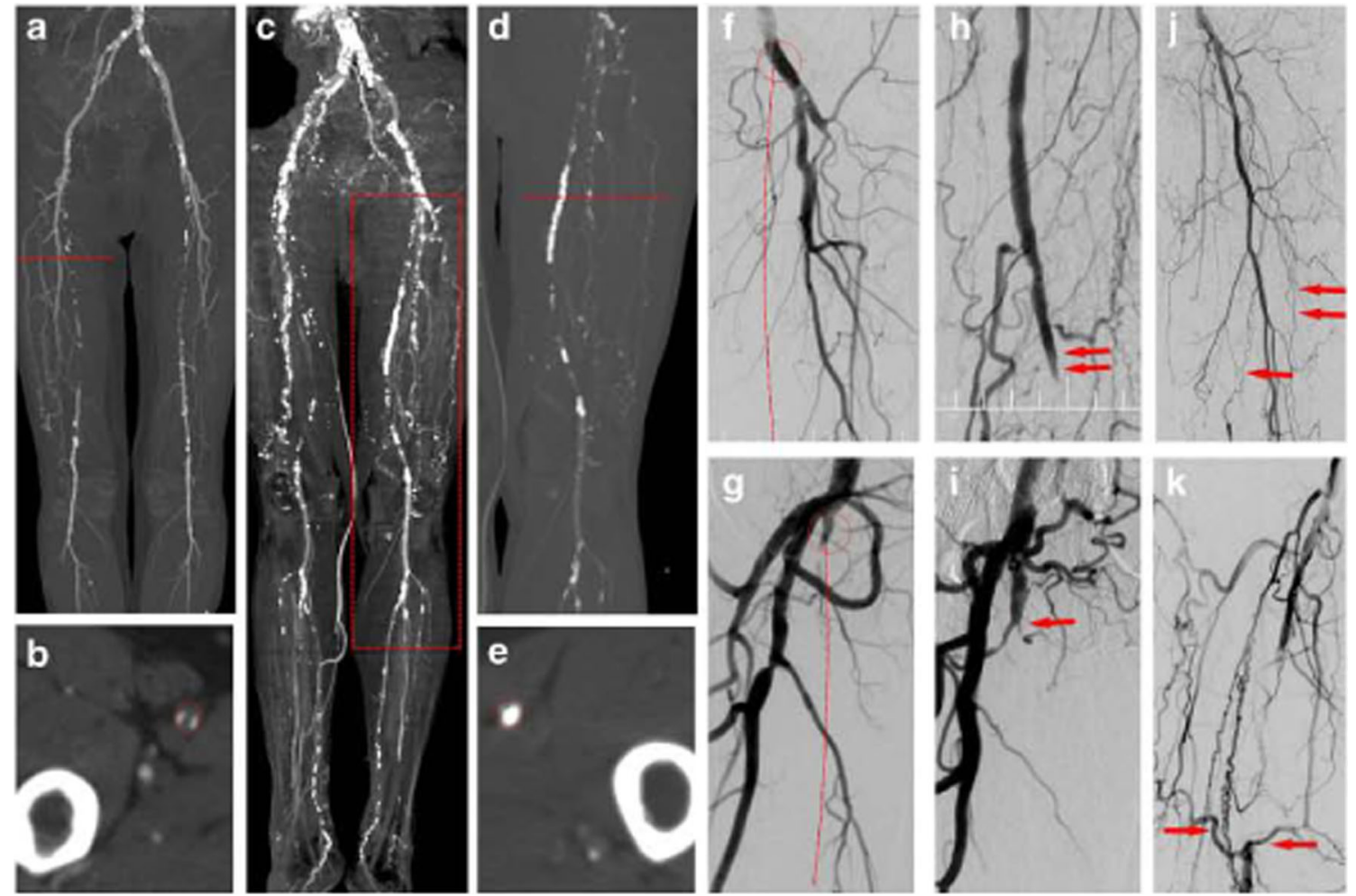

Fig. 2 CT images of representation for qualitative visual assessment of the global amount of calcification $(\mathbf{a}, \mathbf{c})$ and the characterisation of the calcifications based on their axial location (b, e). d Partial highlight of calcification grade 2. Stump morphology was classified as flush (f) or tapered (g) occlusion. Proximal side branches was defined as side branch of greater than $2.0 \mathrm{~mm}$ in size and within $2.0 \mathrm{~cm}$ proximal to the end of

the occlusion. (h referred to no proximal side branch; $\mathbf{i}$ shows existence of proximal side branch). Small collateralisation was defined as circulation in areas occupying less than $25 \%$ of the imaged thigh area and less than $50 \%$ of the diameter of the SFA (j), whereas large collateralisation was defined as circulation areas occupying more than $25 \%$ of the imaged thigh and more than $50 \%$ of the diameter of the SFA (k) 

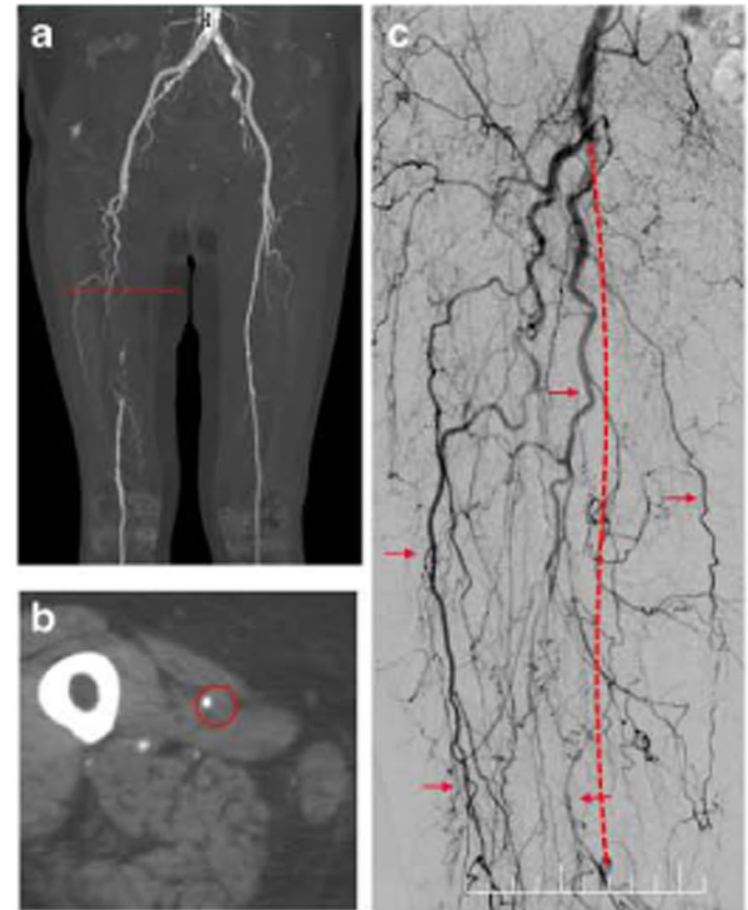

Fig. 3 Antegrade intraluminal recanalisation of a right superficial femoral artery (SFA) chronic total occlusion (CTO). An 83-year-old woman had severe claudication for 2 months and a 20 -year history of diabetes. Lower limb CT angiography (CTA) showed long-segment occlusion in the right SFA accompanied by tapered stub (bold arrow in a),
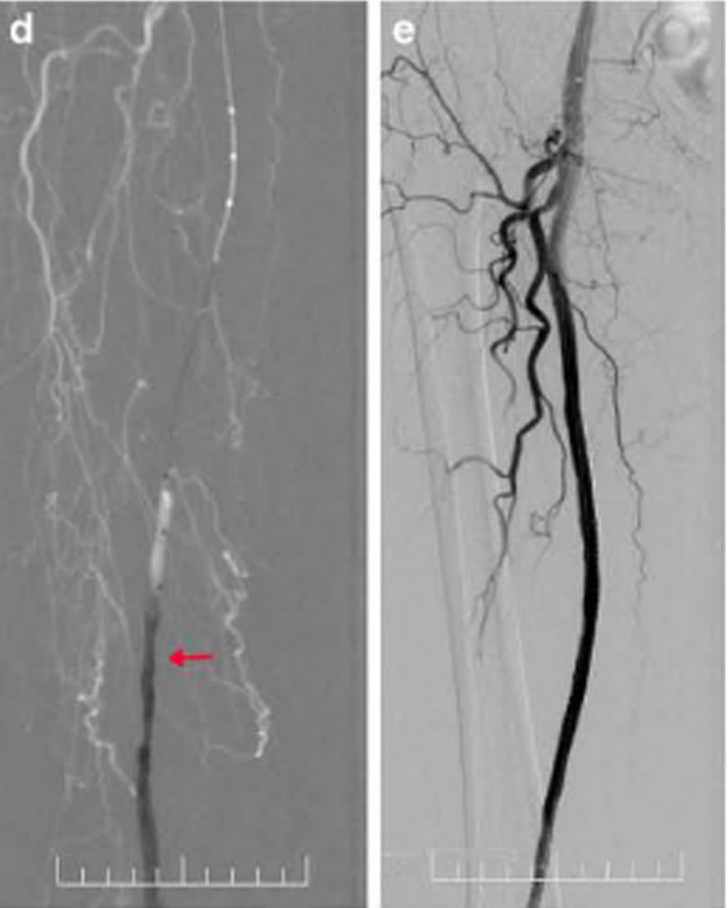

low calcification (circle in b) and small collateral circulation (thin arrows in c). After the antegrade guidewire and catheter passed though the occlusive artery successfully, contrast was injected to confirm the catheter was located in true lumen of distal runoff (d) and recanalisation of right SFA occlusion was achieved (e)
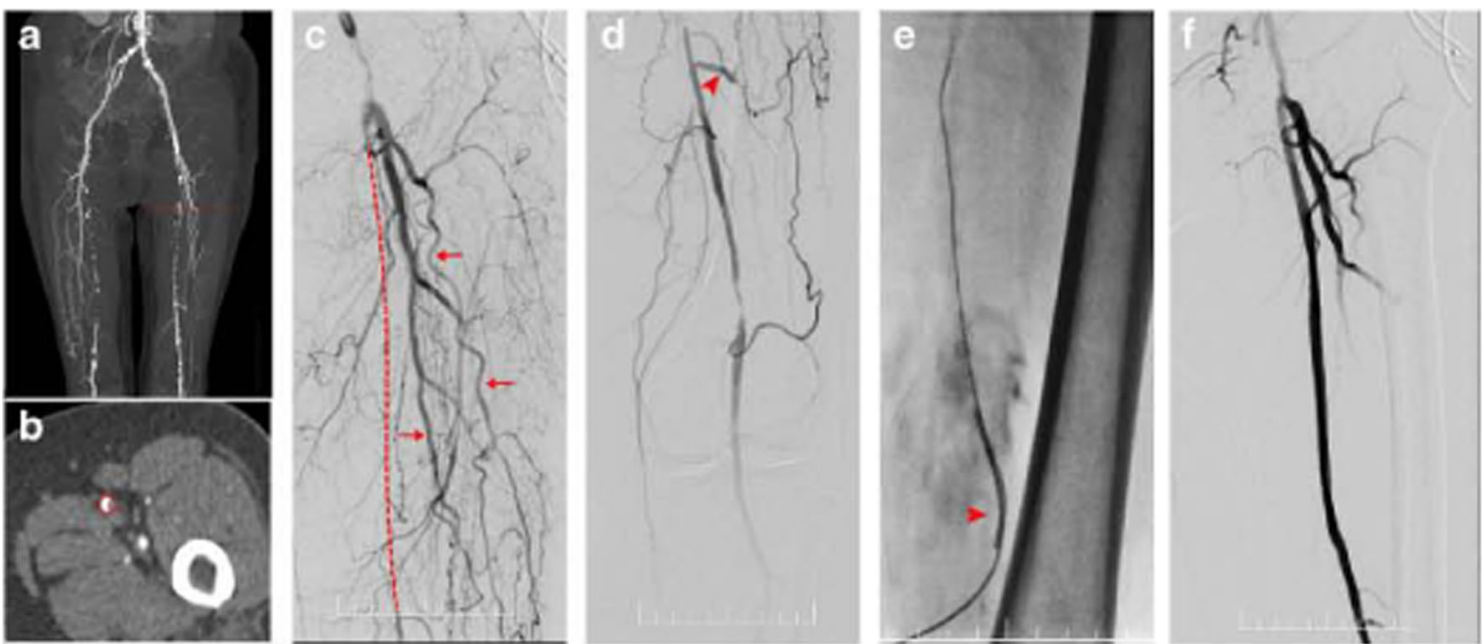

Fig. 4 Retrograde recanalisation of a left superficial femoral artery (SFA) chronic total occlusion (CTO). A 71-year-old woman with an 8-year history of diabetes suffered severe claudication for 15 months. Lower limb CT angiography (CTA) showed long-segment occlusion in the left
SFA accompanied by severe calcification (circle in b), large collateral circulation (thin arrows in $\mathbf{c}$ and arrowhead in d). Successful recanalisation of the SFA was obtained with retrograde puncture in the distal SFA $(\mathbf{e}, \mathbf{f})$ 